\title{
Satisfacción Laboral y Síndrome de Desgaste Laboral en Enfermeros de Hospitales Públicos Córdoba-Argentina
}

\author{
Ob Satisfaction and Professional Wear Syndrome \\ of Nurses Working in Public Hospitals of \\ Córdoba-Argentina
}

\author{
María Sara Díaz Echenique \\ Facultad de Ciencias Médicas-Universidad Nacional \\ de Córdoba. Argentina \\ María Inés Stimolo \\ Facultad de Ciencias Económicas - Universidad Nacional \\ de Córdoba. Córdoba. Argentina \\ Norma Patricia Caro \\ Facultad de Ciencias Económicas - Universidad Nacional \\ de Córdoba. Córdoba. Argentina \\ Recibido: 10-12-09 \\ Aceptado: 14-03-10 \\ Correspondencia: \\ María Inés Stimolo \\ Facultad de Ciencias Económicas-Universidad Nacional de Córdoba \\ Maracaibo 850 Barrio Residencial América \\ 5012 Córdoba. Argentina \\ Télef: +54-035 1-4723787 \\ E-mail:mstimolo@eco.unc.edu.ary mstimolo@gmail.com
}

Resumen

La falta de satisfacción laboral sumada a características propias de cada persona, actuarían como condicionantes para la aparición del Síndrome Burnout (BO), enfermedad laboral que afecta a profesionales expuestos a condiciones psicosociales desfavorables en su medio laboral.

El objetivo de esta investigación fue determinar el nivel de satisfacción laboral, la prevalencia del $\mathrm{BO}$ y su relación con variables socio demográficas, en profesionales de enfermería de hospitales públicos de Córdoba, Argentina, bajo la hipótesis de que existe relación directa entre la falta de satisfacción laboral y BO. Se seleccionó una muestra de enfermeros profesionales, a quienes se les suministró una encuesta socio demográfica, un cuestionario de satisfacción laboral y el Maslach Burnout Inventory.

De un estudio cuantitativo aplicando distintas metodologías estadísticas, como Análisis Factorial de Correspondencias Múltiples (AFCM), Regresión Logística y Contrastes de Independencia para variables ordinales, la población estudiada se clasificó como satisfecha y muy satisfecha, sin embargo, casi un cuarto de la misma padece algún grado de BO. Se establece en esta muestra, un perfil de riesgo para BO o alguno de sus tres componentes, a quienes tienen más de 40 años, no tienen hijos ni pareja, viven en el interior de la Provincia, no están satisfechos laboralmente y trabajan prioritariamente en hospitales provinciales.

Med Segur Trab (Internet) 2010; 56 (218): 22-38

Palabras claves: Burnout-Satisfacción laboral-Regresión Logística - AFCM-Contrastes de Independencia. 


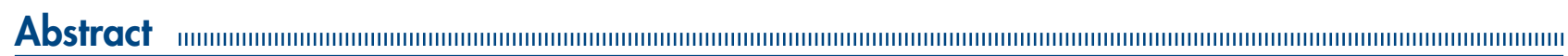

The lack of job satisfaction added each person's own characteristics would function as determining factors in the appearance of the Burnout Syndrome (BO) which is a job illness that affects professionals who are exposed to unfavorable psychosocial conditions in their labor environment.

The aim of this investigation was to determine the level of job dissatisfaction, the prevalence of BO and its relationship with socio demographic variables, in professional nurses working in public hospitals of Cordoba, Argentina, based on the hypothesis that there is a direct relationship between the lack of job satisfaction and BO. Professional nurses were selected and they were provided with a socio demographic survey, a questionnaire of job satisfaction and the Maslach Burnout Inventory.

From a quantitative study applying different statistical methodologies like: Multiple Correspondence Analysis (MCA), Logistic Regression and Contrasts of Independence for ordinary variables, the studied population was classified as satisfied and very satisfied. However, almost a quarter suffer from some grade of $\mathrm{BO}$.

Some BO risk or some of its three components are established to those people who are over 40 years old, without children and live outside the Province. They are not satisfied with their jobs and they work mainly in provincial hospitals.

Med Segur Trab (Internet) 2010; 56 (218): 22-38

Key words: Burnout-Job satisfaction-MCA-Logistic Regression-Contrasts of Independence. 


\section{INTRODUCCIÓN}

El desempeño laboral está determinado por condiciones individuales (edad, sexo, estado civil, capacitación), organizacionales (condiciones de trabajo, seguridad laboral, volumen de trabajo, insumos), del sector salud (reglamentación, relaciones laborales, centralización, roles) y del entorno (crecimiento económico, políticas sociales) (1). Estos determinantes del desempeño laboral actuarían condicionando el bienestar de los trabajadores del sistema de salud y por consiguiente el nivel de satisfacción laboral. Es así que la falta de satisfacción laboral sumada a algunas variables individuales, actuarían como condicionantes para la aparición del Síndrome de Desgaste Laboral o "Burnout" (BO) enfermedad laboral que afecta principalmente a profesionales que brindan servicios. Se define como Burnout(2) a la patología que surge como consecuencia de la exposición de los trabajadores a condiciones psicosociales desfavorables en su medio laboral.

En 1976, Maslach ${ }^{(1)}$ estudió las $\mathrm{r}^{\circ}$ espuestas emocionales de personas que trabajaban en profesiones de ayuda y describió un proceso que denominó "pérdida de responsabilidad profesional". Definió a la enfermedad como "un síndrome de stress crónico en aquellas profesiones de servicios o de ayuda que se caracterizan por una atención intensa y prolongada a personas que están en situación de necesidad o dependencia” y describió en este proceso, tres dimensiones:

- Cansancio emocional: es la primera fase del proceso que consiste en la reducción progresiva de la energía, que se acompaña de la sensación de no poder dar más de si mismo, hay desproporción entre el trabajo realizado y el cansancio que manifiesta que lo torna irritable e incapaz de disfrutar de la tarea que realiza.

- Despersonalización: es el desarrollo de actitudes y sentimientos fríos, negativos, hostiles, hacia los pacientes y colegas. Hay apreciación negativa del propio papel profesional, evitando involucrarse en ello.

- Baja realización personal: pérdida de la confianza en sí mismo, auto valoración negativa, sentimientos de incapacidad junto a la percepción de que no hay posibilidades de crecimiento en el trabajo. Esta es la tercera fase del proceso en la que se produce un alejamiento de la vida familiar, social y recreativa.

La enfermería es reconocida como una profesión estresante. Esto es porque el trabajo del enfermero profesional implica actividades técnicas, relacionales, de organización y administrativas, con grandes responsabilidades, en continuo contacto con la enfermedad, el sufrimiento y la muerte. A lo expuesto, se debe añadir que el trabajo con personas en situaciones de fragilidad genera una gran carga emocional.

A estas características del trabajo hay que sumarle las situaciones de sobrecarga laboral, los conflictos que se producen por la falta de autonomía para tomar decisiones, los salarios bajos, la falta de estímulos y de desarrollo profesional, las condiciones de trabajo, las relaciones con jefes, colegas y compañeros de trabajo, la demanda familiar, ya que es una profesión prioritariamente femenina y en muchas oportunidades desarrollan doble jornada laboral en distintas instituciones de salud. (Jofre y Valenzuela, 2005 (2)).

Estas condiciones de trabajo influyen de manera significativa sobre la satisfacción laboral del enfermero y en consecuencia en la calidad de atención que presta al paciente, a la familia y a la comunidad.

Por otra parte, el Burnout tiene importantes repercusiones socio económicas en términos financieros, tanto por las pérdidas de personal calificado que se producen, como por el costo físico y psíquico que implica para el trabajador.

(1) DUSSAULT Gilles. OPS/OMS $47^{\circ}$ Sesión del Consejo Directivo. 27/09/06.

(2) Denominado también Síndrome de Quemarse por el Trabajo ó Síndrome de Desgaste Profesional ó Síndrome de Desgaste Laboral. 
Ante esta situación, se plantea la necesidad de que las instituciones se preocupen por las condiciones de vida laboral de sus empleados, ya que su salud se manifiesta en la calidad de las prestaciones que brinda.

Además, en contraposición con otras profesiones cuyos riesgos laborales son reconocidos y se previenen con marcos regulatorios legales, existe escasa conciencia preventiva en relación a esta profesión, dándose como implícito que las tareas sanitarias conllevan un riesgo psicosocial que puede afectar a la salud.

Ante el panorama descripto se hace necesario estudiar el síndrome de Burnout, a fin de identificar las condiciones predisponentes o desencadenantes que lo ocasionan y elaborar estrategias de prevención como un derecho básico de protección de la salud de los enfermeros. Por lo que este trabajo tiene como objetivo conocer el nivel de satisfacción laboral y la prevalencia del síndrome de desgaste profesional y su relación con variables socio-demográficas (edad, sexo, estado civil, hijos, nivel de desempeño, antigüedad en la profesión y en el cargo y tipo de administración según dependa el hospital) en una muestra de enfermeros profesionales de hospitales públicos de Córdoba, Argentina, estableciendo como hipótesis del mismo que existe una relación directa entre insatisfacción y síndrome de desgaste laboral.

\section{MATERIAL Y METODOS}

Para la recolección de datos se elaboró una encuesta anónima y autoadministrada, a enfermeros profesionales de hospitales públicos de Córdoba. Autorizaron la administración de la encuesta 13 hospitales, abarcando algo más del 10\% de la dotación de enfermeros profesionales, a los que se les distribuyó 333 encuestas. Esto se llevó a cabo entre el mayo y junio de 2007.

La encuesta, constaba de tres partes. La primera estuvo dirigida a indagar sobre variables socio-demográficas como: edad, sexo, estado civil, número de hijos, domicilio (Córdoba capital o Interior), turno, nivel de desempeño, hospital y antigüedad en la profesión y en el puesto de trabajo. La segunda parte, indagó sobre la satisfacción laboral de los enfermeros, para lo cual se realizó una modificación de la Escala General de Satisfacción diseñada por Warr, Cook y Wall (1979) (3). A través de esta se midió la satisfacción general, intrínseca (SI) (las 7 preguntas pares) y extrínseca (SE) (las 8 preguntas impares) de la teoría bifactorial de Herzberg. Esta escala estuvo constituida por 15 preguntas con 5 opciones de respuesta en una escala Likert. Dichas opciones fueron: "nada" = 1; "poco" = 2; "indiferente" = 3; "moderadamente" = 4 y "en gran medida" = 5. La puntuación total se obtuvo de la suma de los puntos correspondientes al ítem señalado por el profesional encuestado y osciló entre 15 y 75, de manera tal que a mayor puntaje, mayor satisfacción general. Del mismo modo se procedió para la suma de los puntajes de las subescalas que midieron SI (7-35 puntos) y SE (8-40 puntos). La suma de los puntajes asignados a cada opción permitió clasificar a la muestra analizada en 5 grupos que se detallan en la tabla I (a).

Tabla I: Escalas de nivel de satisfacción y dimensiones del síndrome de Burnout

(a) Niveles de satisfacción según puntaje alcanzado en cada escala

\begin{tabular}{lccc}
\hline Niveles de satisfacción & \multicolumn{3}{c}{ Puntajes de cada escala } \\
\hline & general & intrínseca & extrínseca \\
Muy insatisfecho & $1-15$ & $1-7$ & $1-8$ \\
Insatisfecho & $16-30$ & $8-14$ & $9-16$ \\
Indiferente & $31-45$ & $15-21$ & $17-24$ \\
Satisfecho & $46-60$ & $22-28$ & $25-32$ \\
Muy satisfecho & $61-75$ & $29-35$ & $33-40$ \\
\hline
\end{tabular}


Se consideraron las escalas: general, satisfacción intrínsica y satisfacción extrínseca, según Warr, Cook y Wall (1979).

(b) Burnout, niveles según puntaje alcanzado en cada escala

\begin{tabular}{lccc}
\hline $\begin{array}{l}\text { Puntuación } \\
\text { Escala }\end{array}$ & Alta & Media & Baja \\
\hline Cansancio emocional & $\geqq 27$ & $19-26$ & $\leqq 18$ \\
Despersonalización & $\geqq 10$ & $6-9$ & $\leqq 5$ \\
Realización personal & $\geqq 40$ & $34-39$ & $\leqq 33$ \\
\hline
\end{tabular}

Medición de las tres dimensiones del Síndrome según Maslach Burnout Inventory (Maslach y Jackson (1981).

La tercera parte de la encuesta, fue el Maslach Burnout Inventory (Maslach y Jackson, 1981) (4), constituido por 22 ítems en forma de afirmaciones sobre los sentimientos y actitudes del profesional hacia su trabajo y sus pacientes. En dicho instrumento el profesional debió marcar entre 7 opciones de respuesta referidas a con qué frecuencia experimenta los sentimientos que describe cada ítem. Estas respuestas, se valoraron mediante una escala Likert y van desde "nunca" $=0$, "pocas veces al año" $=1$, "una vez al mes o menos" = 2, "unas pocas veces al mes" = 3, "una vez a la semana" = 4, "pocas veces a la semana" $=5 \mathrm{y}$ "todos los días" $=6$.

Este instrumento mide las tres dimensiones del síndrome, cansancio emocional, despersonalización y realización personal. Cada subescala se valora de manera independiente y según el puntaje alcanzado en cada una de ellas, el resultado clasifica como alto, medio o bajo. Los resultados de cada escala no se combinan, por lo tanto a diferencia del instrumento que mide satisfacción, no resulta en una puntuación global de Burnout. (Tabla I (b)).

Después de un análisis descriptivo de las variables de estudio, se analizó por un lado la asociación entre el nivel de satisfacción con el Síndrome de Desgaste Laboral y los componentes del mismo y por otro lado los factores de riesgo del síntoma de desgaste laboral. Las pruebas estadísticas que pretenden probar hipótesis referidas a los patrones de comportamiento de frecuencias relacionadas con variables cualitativas utilizan diferentes estadísticos que proporcionan medidas de asociación cuando las variables se miden en una escala ordinal. Estos estadísticos están basados en el número de concordancias y discordancias que aparecen al comparar las puntuaciones asignadas a los mismos casos según dos variables diferentes..Se utilizaron el Estadístico Gamma, Medida Tau- $b$ de Kendall, Medida Tau-c de Kendall, y el estadístico D de Somers.

Para determinar los factores de riesgo de la satisfacción total, se utilizó un modelo de regresión Logística, recategorizando a la variable satisfacción en dos categorías: No Satisfecho que agrupa los niveles Muy Insatisfecho, Insatisfecho e Indiferente y Satisfecho que agrupa los niveles Satisfecho y Muy satisfecho. En el procesamiento se incluyeron 221 empleados ya que seis casos fueron eliminados por tener datos faltantes; las variables predictoras significativas estadísticamente en el modelo fueron convenientemente categorizadas.

Esta metodología también fue utilizada para determinar los factores de riesgo de tener BO y para cada una de las dimensiones del síndrome, considerando las variables socioeconómicas como predictoras.

Se utilizó el Análisis Factorial de Correspondencias Múltiples (AFCM) para evaluar la relación entre las variables sociodemográficas y las dimensiones del Burnout, detectando empleados con características similares. 
A partir de los ejes factoriales del AFCM, para completar el estudio se realizó un Análisis de Clasificación con el fin de realizar un agrupamiento de los empleados con características similares

\section{RESULTADOS}

De un primer análisis de los datos resultó que del total de encuestados:

- respondieron $68.1 \%$

- $81.9 \%$ fueron mujeres,

- edad promedio: 40.7 años,

- 47.1\% está en pareja,

- $61.2 \%$ tiene hijos,

- 82.6\% es de Córdoba Capital,

- $67.4 \%$ trabajan en hospitales provinciales, $19.8 \%$ municipales y $17.7 \%$ universitarios,

- antigüedad media de 15.6 años en la profesión y 10.9 años en el puesto de trabajo,

- No hubo enfermeros muy insatisfechos.

La media aritmética y los desvíos estándar para Satisfacción Total, Satisfacción Intrínseca y Satisfacción Extrínseca fueron 48.9 (DS 11.1); 22.62 (DS 6.31) y 26.44 (DS 5.48), correspondiendo a la categoría de satisfecho en todos los casos.

Para Burnout, la variable más afectada fue Cansancio Emocional, luego Realización Personal y finalmente Despersonalización cuyos puntajes promedios fueron 23.34, 37.15 y 6.63 respectivamente, correspondiendo a un nivel medio en todos los casos.

Considerando los 53 empleados que resultaron con Síndrome de desgaste laboral se considera la relación entre Satisfacción total, intrínseca y extrínseca, con el nivel de Desgaste Laboral. Las medidas de asociación indican que a mayor nivel de satisfacción, menor es el grado de desgaste laboral. La medida Tau-b de Kendall nos proporciona un valor de -0,48 (Tabla II a), lo cual significa un moderado grado de asociación negativa. Estas conclusiones coinciden cuando se toman por separado la satisfacción intrínseca y extrínseca (Tabla II b y c). 
Tabla II: Asociación entre el Desgaste Laboral y Satisfacción

(a) Tabla satisfacción total según Desgaste Laboral y Medidas simétricas y direccionales

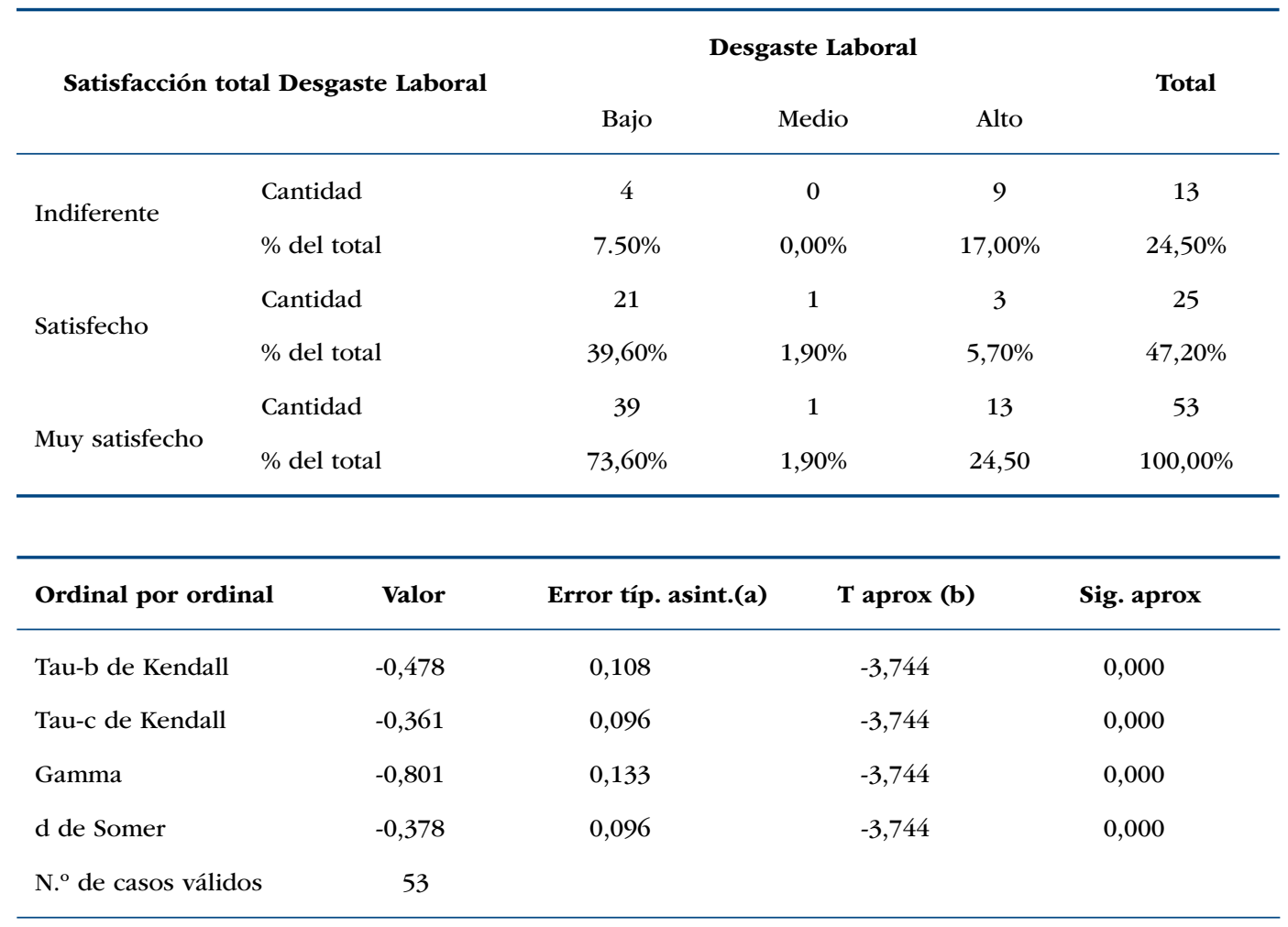

(a) Asumiendo la hipótesis alternativa.

(b) Empleando el error típico asintótico basado en la hipótesis nula.

(b) Tabla satisfacción intrinseca según Desgaste Laboral y Medidas simétricas y direccionales

\begin{tabular}{|c|c|c|c|c|c|}
\hline \multicolumn{2}{|c|}{ Satisfacción intrínsica } & \multicolumn{3}{|c|}{ Desgaste Laboral } & Total \\
\hline \multirow{2}{*}{ Insatisfecho } & Cantidad & 0 & 0 & 3 & 3 \\
\hline & $\%$ del total & $0,00 \%$ & $0,00 \%$ & $5,70 \%$ & $5,70 \%$ \\
\hline \multirow{2}{*}{ Indiferente } & Cantidad & 6 & 0 & 6 & 12 \\
\hline & $\%$ del total & $11,30 \%$ & $0,00 \%$ & $11,30 \%$ & $22,60 \%$ \\
\hline \multirow{2}{*}{ Satisfecho } & Cantidad & 17 & 1 & 4 & 22 \\
\hline & $\%$ del total & $32,10 \%$ & $1,90 \%$ & $7,50 \%$ & $41,50 \%$ \\
\hline \multirow{2}{*}{ Muy satisfecho } & Cantidad & 16 & 0 & 0 & 16 \\
\hline & $\%$ del total & $30,20 \%$ & $0,00 \%$ & $0,00 \%$ & $30,20 \%$ \\
\hline \multirow{2}{*}{ Total } & Recuento & 39 & 1 & 13 & 53 \\
\hline & $\%$ del total & $73,60 \%$ & $1,90 \%$ & $24,50 \%$ & $100,00 \%$ \\
\hline
\end{tabular}




\begin{tabular}{|c|c|c|c|c|}
\hline Ordinal por ordinal & Valor & Error típ. asint.(a) & $\mathbf{T}$ aprox (b) & Sig. aprox \\
\hline Tau-b de Kendall & $-0,511$ & 0,082 & $-4,647$ & 0,000 \\
\hline Tau-c de Kendall & $-0,399$ & 0,086 & 4,647 & 0,000 \\
\hline Gamma & $-0,862$ & 0,079 & $-4,647$ & 0,000 \\
\hline d de Somer & $-0,39$ & 0,077 & $-4,647$ & 0,000 \\
\hline N. ${ }^{\circ}$ de casos válidos & 53 & & & \\
\hline
\end{tabular}

(a) Asumiendo la hipótesis alternativa.

(b) Empleando el error típico asintótico basado en la hipótesis nula

(c) Tabla satisfacción extrínseca según Desgaste Laboral y Medidas simétricas y direccionales.

\begin{tabular}{llcccc}
\hline \multirow{2}{*}{ Satisfacción extrínsica } & \multicolumn{3}{c}{ Desgaste Laboral } & & Total \\
& & Bajo & Medio & Alto & \\
\hline \multirow{2}{*}{ Insatisfecho } & Cantidad & 0 & 0 & 1 & 1 \\
& \% del total & $0,00 \%$ & $0,00 \%$ & $1,90 \%$ & $1,90 \%$ \\
\multirow{2}{*}{ Indiferente } & Cantidad & 5 & 0 & 7 & 12 \\
& \% del total & $9,40 \%$ & $0,00 \%$ & $13,20 \%$ & $22,60 \%$ \\
\multirow{2}{*}{ Satisfecho } & Cantidad & 23 & 1 & 4 & 28 \\
& \% del total & $43,40 \%$ & $1,90 \%$ & $7,50 \%$ & $52,80 \%$ \\
Muy satisfecho & Cantidad & 11 & 0 & 1 & 12 \\
& \% del total & $20,80 \%$ & $0,00 \%$ & $1,90 \%$ & $22,60 \%$ \\
& Recuento & 39 & 1 & 13 & 53 \\
\hline
\end{tabular}

\begin{tabular}{lcccc}
\hline Ordinal por ordinal & Valor & Error típ. asint.(a) & T aprox (b) & Sig. aprox \\
\hline Tau-b de Kendall & $-0,411$ & 0,115 & $-3,146$ & 0,002 \\
Tau-c de Kendall & $-0,305$ & 0,097 & $-3,146$ & 0,002 \\
Gamma & $-0,726$ & 0,162 & $-3,146$ & 0,002 \\
d de Somer & $-0,329$ & 0,099 & $-3,146$ & 0,002 \\
N. ${ }^{\circ}$ de casos válidos & 53 & & & \\
\hline
\end{tabular}

(a) Asumiendo la hipótesis alternativa.

(b) Empleando el error típico asintótico basado en la hipótesis nula.

Luego se corrobora la hipótesis de dependencia entre Nivel de Satisfacción (total, intrínseca y extrínseca) y cada componente del Síndrome de Desgaste.

Considerando los componentes del Síndrome de Desgaste Laboral, despersonalización, cansancio y realización personal, existe un grado de asociación negativa entre la satisfacción total y el cansancio laboral, a menor nivel de satisfacción mayor cansancio (Tabla III a, Tau-c de Kendall: - 0,30). Lo mismo se observa con la despersonalización, cuyo valor es un poco más bajo (Tabla III b, Tau-c de Kendall: - 0,20). 
Tabla III: Asociación entre Satisfacción Total y componentes del Cansancio Laboral

(a) Tabla de satisf total según Cansancio Laboral y Medidas simétricas y direccionales

\begin{tabular}{|c|c|c|c|c|c|c|}
\hline \multicolumn{2}{|c|}{ Satisfacción total } & \multicolumn{5}{|c|}{ Desgaste Laboral } \\
\hline \multirow{2}{*}{ Insatisfecho } & Cantidad & & 0 & 4 & 8 & 12 \\
\hline & $\%$ del total & & $0,00 \%$ & $1,80 \%$ & $3,60 \%$ & $5,30 \%$ \\
\hline \multirow{2}{*}{ Indiferente } & Cantidad & & 15 & 17 & 42 & 74 \\
\hline & $\%$ del total & & $6,70 \%$ & $7,60 \%$ & $18,70 \%$ & $32,90 \%$ \\
\hline \multirow{2}{*}{ Satisfecho } & Cantidad & & 47 & 26 & 29 & 102 \\
\hline & $\%$ del total & & $20,90 \%$ & $11,60 \%$ & $12,90 \%$ & $45,30 \%$ \\
\hline \multirow[b]{2}{*}{ Muy satisfecho } & Cantidad & & 21 & 7 & 9 & 37 \\
\hline & $\%$ del total & & $9,30 \%$ & $3,10 \%$ & $4,00 \%$ & $16,40 \%$ \\
\hline \multirow[b]{2}{*}{ Total } & Recuento & & 83 & 54 & 88 & 225 \\
\hline & $\%$ del total & & $36,90 \%$ & $24,00 \%$ & $39,10 \%$ & $100,00 \%$ \\
\hline \multicolumn{2}{|l|}{ Ordinal por ordinal } & Valor & \multicolumn{2}{|c|}{ Error típ. asint.(a) } & $T$ aprox (b) & Sig. aprox \\
\hline \multicolumn{2}{|l|}{ Tau-b de Kendall } & $-0,31$ & \multicolumn{2}{|c|}{0,053} & $-5,783$ & 0 \\
\hline \multicolumn{2}{|l|}{ Tau-c de Kendall } & $-0,304$ & \multicolumn{2}{|c|}{0,053} & $-3,744$ & 0 \\
\hline \multicolumn{2}{|l|}{ Gamma } & $-0,46$ & \multicolumn{2}{|c|}{0,075} & $-3,744$ & 0 \\
\hline \multicolumn{2}{|l|}{ d de Somer } & $-0,309$ & \multicolumn{2}{|c|}{0,053} & $-3,744$ & 0 \\
\hline N. ${ }^{\circ}$ de casos válidos & & 225 & & & & \\
\hline
\end{tabular}

(b) Tabla de satisf total según Despersonalizacion y Medidas simétricas y direccionales

\begin{tabular}{|c|c|c|c|c|c|}
\hline \multicolumn{2}{|c|}{ Satisfacción total } & \multicolumn{3}{|c|}{ Despersonalización } & Total \\
\hline \multirow{2}{*}{ Insatisfecho } & Cantidad & 5 & 3 & 4 & 12 \\
\hline & $\%$ del total & $2,20 \%$ & $1,30 \%$ & $1,80 \%$ & $5,30 \%$ \\
\hline \multirow{2}{*}{ Indiferente } & Cantidad & 22 & 25 & 27 & 74 \\
\hline & $\%$ del total & $9,80 \%$ & $11,10 \%$ & $12,00 \%$ & $32,90 \%$ \\
\hline \multirow{2}{*}{ Satisfecho } & Cantidad & 53 & 24 & 25 & 102 \\
\hline & $\%$ del total & $23,60 \%$ & $10,70 \%$ & $11,10 \%$ & $45,30 \%$ \\
\hline \multirow[b]{2}{*}{ Muy satisfecho } & Cantidad & 25 & 5 & 7 & 37 \\
\hline & $\%$ del total & $2,20 \%$ & $3,10 \%$ & $16,40 \%$ & \\
\hline \multirow[b]{2}{*}{ Total } & Recuento & 105 & 57 & 63 & 225 \\
\hline & $\%$ del total & $46,70 \%$ & $25,30 \%$ & $28,00 \%$ & $100,00 \%$ \\
\hline
\end{tabular}




\begin{tabular}{lcccc}
\hline Ordinal por ordinal & Valor & Error típ. asint.(a) & T aprox (b) & Sig. aprox \\
\hline Tau-b de Kendall & $-0,208$ & 0,058 & $-3,609$ & 0 \\
Tau-c de Kendall & $-0,202$ & 0,056 & $-3,609$ & 0 \\
Gamma & $-0,317$ & 0,086 & $-3,609$ & 0 \\
d de Somer & $-0,206$ & 0,057 & $-3,609$ & 0 \\
N. ${ }^{\circ}$ de casos válidos & 225 & & & \\
\hline
\end{tabular}

(c) Tabla de satisf total según Realización Personal y Medidas simétricas y direccionales

\begin{tabular}{llcccc}
\hline \multirow{2}{*}{ Satisfacción total } & \multicolumn{3}{c}{ Realización Personal } & \\
& & Bajo & Medio & Alto & Total \\
\hline \multirow{2}{*}{ Insatisfecho } & Cantidad & 9 & 2 & 1 & 12 \\
& \% del total & $4,00 \%$ & $0,90 \%$ & $0,40 \%$ & $5,30 \%$ \\
\multirow{2}{*}{ Indiferente } & Cantidad & 37 & 21 & 16 & 74 \\
& \% del total & $16,40 \%$ & $9,30 \%$ & $7,10 \%$ & $32,90 \%$ \\
\multirow{3}{*}{ Satisfecho } & Cantidad & 18 & 19 & 65 & 102 \\
& \% del total & $8,00 \%$ & $8,40 \%$ & $28,90 \%$ & $45,30 \%$ \\
Total & Cantidad & 3 & 6 & 28 & 37 \\
& \% del total & $1,30 \%$ & $2,70 \%$ & $12,40 \%$ & $16,40 \%$ \\
\hline
\end{tabular}

\begin{tabular}{lcccc}
\hline Ordinal por ordinal & Valor & Error típ. asint.(a) & T aprox (b) & Sig. aprox \\
\hline Tau-b de Kendall & 0,433 & 0,048 & 8,742 & 0 \\
Tau-c de Kendall & 0,417 & 0,048 & 8,742 & 0 \\
Gamma & 0,633 & 0,063 & 8,742 & 0 \\
d de Somer & 0,424 & 0,047 & 8,742 & 0 \\
N.o de casos válidos & 225 & & & \\
\hline
\end{tabular}

La despersonalización y el Cansancio laboral dependen del nivel de satisfacción, y en ambos casos la asociación es negativa. Estas mismas conclusiones se obtienen cuando se analiza la satisfacción intrínseca y extrínseca.

A diferencia de los dos componentes anteriores analizados, la Realización personal tiene una relación directa con el grado de satisfacción (Tabla III c). Estas conclusiones también se obtienen cuando se analiza la satisfacción intrínseca y extrínseca.

A través del modelo de regresión logística se determinaron, en primer lugar los factores de riesgo que afectan a la Satisfacción Total. Resultaron estadísticamente significativas (Tabla IV a) a un nivel de significación de 0,10 , ciudad del hospital y turno de trabajo. De ello, se puede concluir que:

- Los empleados de hospitales del Interior de Córdoba tienen una chance 6 veces mayor de estar insatisfechos que los enfermeros que pertenecen a los hospitales de la Ciudad de Córdoba. 
- Los enfermeros del turno noche tienen una chance 1,4 veces mayor de no estar satisfechos que los enfermeros del turno de la mañana.

Los factores de riesgo para estar insatisfecho son trabajar en el turno noche y en hospitales del Interior Provincial.

Tabla IV: Resultados de las regresiones logísticas para SatisfacciónTotal y Síndrome de Quemado

(a) Regresión logística para explicar Satisfacción Total

Codificación de la variable dependiente

\begin{tabular}{|c|c|c|c|c|c|c|}
\hline \multicolumn{4}{|c|}{ Valor original } & \multicolumn{3}{|c|}{ Valor interno } \\
\hline \multicolumn{4}{|c|}{ Satisfecho } & \multicolumn{2}{|r|}{0} & \\
\hline \multicolumn{4}{|c|}{ No satisfecho } & \multicolumn{2}{|r|}{1} & \\
\hline \multicolumn{7}{|c|}{ Codificaciones de variables categóricas } \\
\hline & & & \multirow{2}{*}{\multicolumn{2}{|c|}{$\begin{array}{c}\text { Frecuencia } \\
\text { (1) }\end{array}$}} & \multicolumn{2}{|c|}{ Codificación de parámetros } \\
\hline & & & & & (2) & (1) \\
\hline \multirow{3}{*}{ Turno } & \multicolumn{2}{|c|}{ Mañana/Tarde } & \multicolumn{2}{|c|}{158} & 0,000 & 0,000 \\
\hline & \multicolumn{2}{|c|}{ Noche } & \multicolumn{2}{|c|}{30} & 1,000 & 0,000 \\
\hline & \multicolumn{2}{|c|}{ Rotativo } & \multicolumn{2}{|c|}{34} & 0,000 & 1,000 \\
\hline \multirow{2}{*}{ Ciudad } & \multicolumn{2}{|c|}{ Ciudad de Córdoba } & \multicolumn{2}{|c|}{188} & \multicolumn{2}{|l|}{0,000} \\
\hline & \multicolumn{2}{|c|}{ Interior de Córdoba } & & & \multicolumn{2}{|l|}{1,000} \\
\hline \multicolumn{7}{|c|}{ Variables en la ecuación } \\
\hline & $\mathbf{B}$ & E.T. & Wald & gl & Sig. & $\operatorname{Exp}(B)$ \\
\hline $\begin{array}{l}\text { r_ciudad } \\
\text { (Interior) }\end{array}$ & 2,063 & 0,474 & 18,909 & 1 & 0,000 & 7,867 \\
\hline r_turno & & & 4,596 & 2 & 0,100 & \\
\hline $\begin{array}{l}\text { r_turno } \\
\text { (Noche) }\end{array}$ & 0,876 & 0,420 & 4,346 & 1 & 0,037 & 2,401 \\
\hline $\begin{array}{l}\text { r_turno } \\
\text { (Rotativo) }\end{array}$ & 0,369 & 0,444 & 0,690 & 1 & 0,406 & 1,446 \\
\hline Constante & $-0,955$ & 0,183 & 27,218 & 1 & 0,000 & 0,385 \\
\hline
\end{tabular}

(a) a Variable(s) introducida(s) en el paso 1: r_ciudad, r_turno.

(b) Regresión logística para explicar Síndrome de Quemado

\begin{tabular}{llcc}
\hline & Variables Predictoras & Frecuencia & $\begin{array}{c}\text { Codificación de } \\
\text { parámetros }\end{array}$ \\
\hline $\begin{array}{l}\text { Edad } \\
\text { (REDAD) }\end{array}$ & 1. Hasta 40 años & 97 & 1,000 \\
& 2. Más de 40 años & 111 & 0,000 \\
$\begin{array}{l}\text { Estado Civil } \\
\text { (RESTCIV) }\end{array}$ & 1. Soltero, separado, divorciado o viudo & 107 & 1,000 \\
& 2. Casado o en pareja & 101 & 0,000 \\
\hline
\end{tabular}

a Variable(s) introducida(s) en el paso 1: ANTIGUED, RESTCIV, REDAD. 
En segundo lugar, se analizaron los factores de riesgo que afectan al BO.

Considerando todos los empleados, en total 208 casos con las respuestas completas, se realizó una regresión logística. La variable dependiente está codificada como 0: sano y 1: quemado, y las variables independientes que resultaron significativas (Tabla IV b) fueron edad y estado civil. Los coeficientes de la regresión que se obtuvieron, permiten concluir que:

- Los empleados que no están en pareja tienen un 92,6\% más de probabilidad de presentar el síndrome de Burnout que los empleados que están en pareja.

- Los empleados menores de 40 años tienen una chance del 57\% menor que los que tienen más de esa edad de presentar este síndrome.

En resumen los factores de riesgo resultan ser, estar sin pareja y ser mayor de 40 años.

En tercer lugar, para analizar los factores de riesgo para cada una de las dimensiones del síndrome de Desgaste Laboral, se consideró, una regresión logística para cada una.

Para cansancio emocional, la categoría de referencia de la variable agrupó los niveles medio y alto. Del análisis de la Tabla V (a), se puede concluir que:

- La antigüedad en el trabajo no tiene efecto sobre la probabilidad de que una persona presente cansancio emocional.

- Los empleados sin hijos tienen una probabilidad de tener cansancio emocional 2,4 veces mayor que los que tienen hijos.

- Los empleados que están satisfechos tienen un $77,7 \%$ menor de chance de tener cansancio emocional que los que no están satisfechos.

En resumen los factores de riesgo son: no tener hijos y estar insatisfechos.

Tabla V: Resultados de las regresiones logísticas para las componentes del Síndrome de Quemado (a) Variables predictoras y Variables en la ecuación (Cansancio Emocional)

\begin{tabular}{llcc}
\hline & Variables Predictoras & Frecuencia & $\begin{array}{c}\text { Codificación de } \\
\text { parámetros }\end{array}$ \\
\hline Satisfacción total & 1. Satisfecho & 109 & 1 \\
Hijos & 2. No satisfecho & 71 & 0 \\
(RHIJOS) & 1. Sin hijos & 53 & 1 \\
\hline
\end{tabular}

\begin{tabular}{lcccccc}
\hline Variables & B & E.T. & Wald & gl & Sig. & Exp(B) \\
\hline ANTIGUED & 0,063 & 0,021 & 8,583 & 1 & 0,003 & 1,065 \\
RHIJOS (sin hijos) & 0,871 & 0,400 & 4,735 & 1 & 0,030 & 2,389 \\
Satisfacción total (satisfecho) & $-1,498$ & 0,344 & 18,935 & 1 & 0,000 & 0,223 \\
Constante & $-0,523$ & 0,360 & 2,114 & 1 & 0,146 & 0,593 \\
\hline
\end{tabular}

(b) Variables en la ecuación (Despersonalización)

\begin{tabular}{lcccccc}
\hline \multicolumn{1}{c}{ Variables } & B & E.T. & Wald & gl & Sig. & Exp(B) \\
\hline Satisfacción Total (Satisfecho) & $-0,623$ & 0,330 & 3,556 & 1 & 0,059 & 0,537 \\
Constante & $-0,488$ & 0,244 & 3,990 & 1 & 0,046 & 0,614 \\
\hline
\end{tabular}


(c) Variables predictoras y Variables en la ecuación (Realización Personal)

\begin{tabular}{ccccc}
\hline & Variables & Frecuencia & \multicolumn{2}{c}{ Codificación de parámetros } \\
\hline $\begin{array}{c}\text { Hospital en el } \\
\text { cual trabaja } \\
\text { (RHOSPITA) }\end{array}$ & Mrovincial & 128 & $\mathbf{( 1 )}$ & (2) \\
\hline $\begin{array}{c}\text { Satisfacción } \\
\text { total }\end{array}$ & Mnivicipal & 31 & 1,000 & 0,000 \\
Ciudad & Satisfecho & 21 & 0,000 & 1,000 \\
$($ RCIUDAD) & No satisfecho & 109 & 0,000 & 0,000 \\
\hline
\end{tabular}

\begin{tabular}{lcccccc}
\hline Variables & B & E.T. & Wald & gl & Sig. & Exp(B) \\
\hline RCIUDAD (Córdoba) & 1,520 & 0,677 & 5,038 & 1 & 0,025 & 4,572 \\
RHOSPITA & & & 5,043 & 2 & 0,080 & \\
RHOSPITA (Provincial) & $-0,929$ & 0,555 & 2,799 & 1 & 0,094 & 0,395 \\
RHOSPITA (Municipal) & $-0,116$ & 0,663 & 0,030 & 1 & 0,862 & 0,891 \\
Satisfacción total (Satisfecho) & 1,623 & 0,374 & 18,830 & 1 & 0,000 & 5,068 \\
Constante & $-1,758$ & 0,855 & 4,224 & 1 & 0,040 & 0,172 \\
\hline
\end{tabular}

Para la dimensión despersonalización la única variable que resultó significativa es la satisfacción total (Tabla $\mathrm{V}$ b), siendo la probabilidad de despersonalización en los empleados satisfechos un $46,3 \%$ menor que en los empleados insatisfechos. Por lo que la insatisfacción es un factor de riesgo del sentimiento de despersonalización.

Por último, los factores que afectan la Realización Personal, considerando los niveles medio y alto versus el bajo puede analizarse (Tabla V c) que:

- Los empleados que trabajan en la Ciudad de Córdoba tienen 4,6 veces más chance de tener realización personal que los que trabajan en el interior de la Provincia de Córdoba.

- Los que trabajan en hospitales Provinciales y Municipales tienen un 60,5\% y un $10,5 \%$ menos de probabilidad de tener alta realización personal que los que trabajan en hospitales Universitarios.

- Los empleados que están satisfechos tienen una chance 5 veces mayor de sentir realización personal que los que están insatisfechos.

Resumiendo los factores de riesgo para la realización personal son: trabajar en hospitales del Interior de Córdoba, en mayor medida en Hospitales Provinciales, luego en Hospitales Municipales y estar insatisfechos.

En el análisis Factorial de Correspondencias Múltiples (AFCM) fueron consideradas las variables socioeconómicas para encontrar los factores, y los componentes del síndrome de Burnout se incluyeron como variables ilustrativas (Tabla XIV), ya que las mismas se proyectan sobre los ejes factoriales pero no son consideradas en su cálculo. 
Las variables socioeconómicas consideradas en el AFCM, con detalle de código y las categorías consideradas fueron:

\begin{tabular}{ll}
\hline Variable (código ) & Categorías \\
\hline Edad (redad) & - Hasta 40 años \\
& -Más de 40 años \\
Ciudad (rciudad) & -Ciudad de Córdoba \\
& -Interior de Córdoba \\
Turno (rturno) & -Mañana o tarde \\
& -Noche \\
Nivel de desempeño (niveldesem) & -Rotativo \\
& -Operativo \\
& -Conducción \\
Hospital (rhospita) & -Ambos \\
& -Universitario \\
Estado Civil (restciv) & -Provincial \\
& -Municipal \\
& -Casado o en pareja \\
\hline
\end{tabular}

El primer eje factorial (3) (eje horizontal de la Figura 1) está representado por las variables ciudad, el turno y el hospital donde trabaja. A la izquierda se ubican las personas del interior de córdoba, que trabajan en turnos rotativos y en hospitales provinciales. Son quienes poseen un nivel de realización baja y un nivel medio de cansancio emocional. A la derecha están quienes son de la ciudad de Córdoba, trabajan en los turnos mañana o tarde y en hospitales universitarios. Son quienes tienen un alto nivel de realización.

El segundo eje factorial (eje vertical de la Figura 1) está caracterizado por el nivel de desempeño, la edad y el estado civil. Hacia arriba están quienes tienen menos de 40 años, sin pareja y se desempeñan en los niveles operativos. Hacia abajo encontramos los mayores de 40 años, con pareja, que se desempeñan en los niveles de conducción. No resultaron significativas las variables relacionadas con el cansancio y la despersonalización.

Figura 1. Variables y Grupos en los dos primeros Ejes factoriales del AFCM

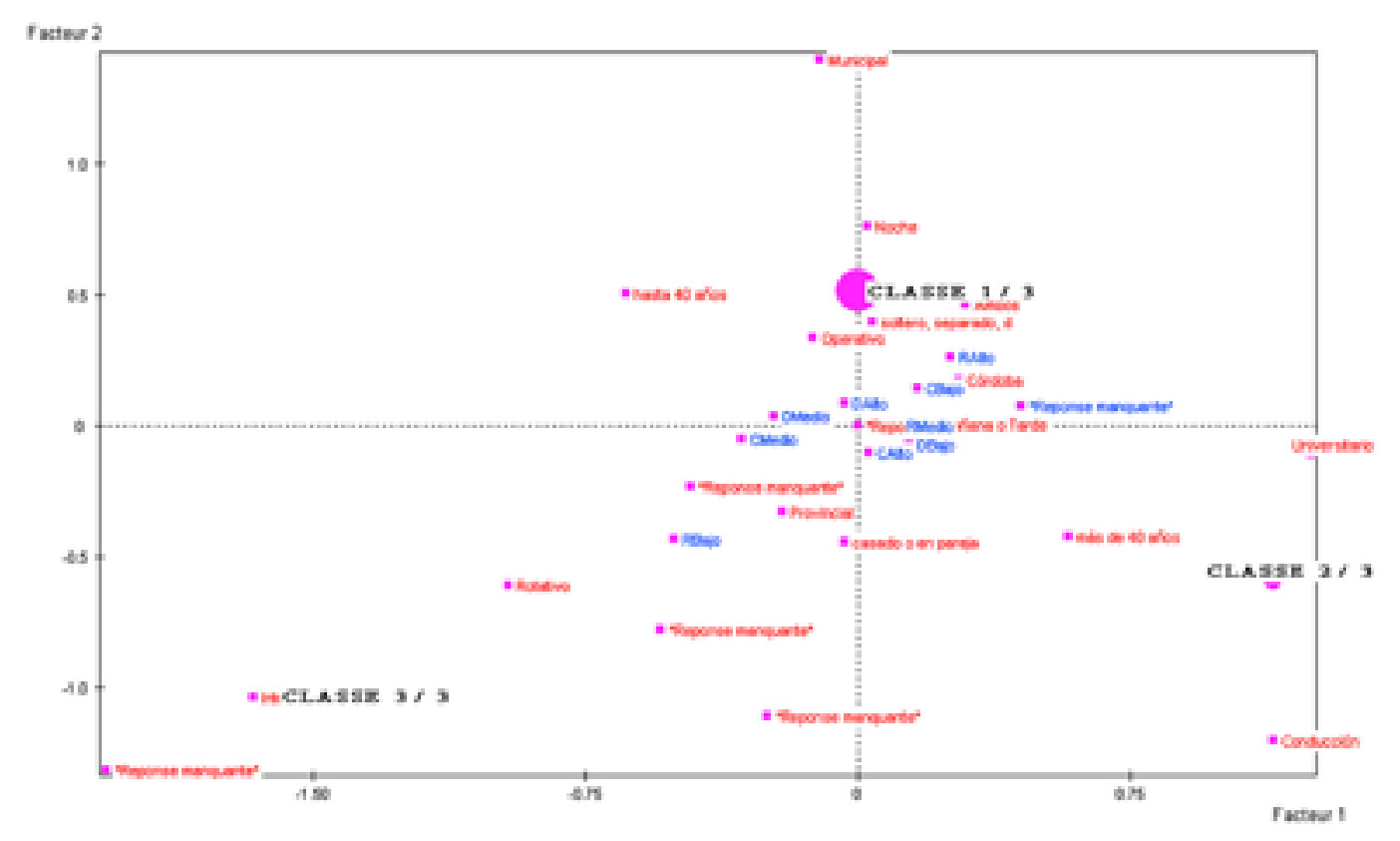

(3) Es el que tiene mayor peso medido en términos del porcentaje de variabilidad total explicada. 
Del Análisis de Clasificación los empleados se agruparon en tres clases, con las siguientes características:

Clase1(60 enfermeros), pertenecen al nivel operativo, de la ciudad de Córdoba, menores de 40 años, que trabajan en el turno de la noche, con alto nivel de realización y bajo cansancio.

Clase2 (23 enfermeros), trabajan en hospitales universitarios, en el nivel de conducción, más de 40 años. No presentan ninguna característica respecto a las dimensiones del desgaste laboral.

Clase3 (17 enfermeros), son del interior, trabajan en turnos rotativos, con nivel bajo de realización.

En la Figura 1, puede observarse gráficamente en los dos primeros ejes factoriales donde se encuentran ubicados los grupos definidos.

\section{DISCUSIÓN Y CONCLUSIONES FINALES}

La tasa de respuesta en esta investigación engloba amplias variaciones entre hospitales (48-96\%), con valores similares a los que se describen en distintos estudios realizados sobre el mismo tema (Atance Martinez. J, 1997; Del Campo, M., FernándezRepeto Valls et al, 1999; Grau, A et al,2006; López, F et al, 2005) (5,6,7,8,)

También, muestra el interés que suscita el tema y la preocupación de las jefaturas y supervisiones de enfermería por la salud y bienestar de las profesionales a su cargo.

Es necesario considerar que puede haber algún sesgo en los resultados, ya que es probable que los profesionales menos satisfechos o con niveles más altos de desgaste no hayan querido participar en esta investigación y responder la encuesta, se encontraran con licencia por enfermedad o hubieran dejado su puesto laboral. Probablemente, por estas mismas razones, ningún profesional clasificó como muy insatisfecho en ninguno de los tres aspectos de la encuesta utilizada.

La única variable socio demográfica que resultó significativa para la falta de satisfacción laboral fue la procedencia (interior de la provincia de Córdoba) de los profesionales. Esto probablemente se deba al mayor esfuerzo en términos de tiempo y dinero que deben realizar cotidianamente para concurrir a trabajar.

Los ítems en los que se registró mayor falta de satisfacción (tanto intrínseca como extrínseca) son aquellos que de alguna manera evalúan cómo perciben los trabajadores sus posibilidades de crecimiento tanto en la institución como en lo económico. Así, una importante proporción de profesionales encuestados afronta su tarea diaria con la percepción de que no recibe un salario adecuado ni tiene posibilidades de ascender en el lugar en el que presta servicio.

A esto, hay que sumarle dos hechos que contribuyen a disminuir la autoestima de las profesionales, sentir que sus opiniones no son tenidas en cuenta por sus compañeros y que el trabajo realizado no es reconocido por las autoridades de salud.

Finalmente, la falta de normas claras de trabajo e igualmente la falta de buena comunicación entre directivos y el nivel operativo son condiciones que producen confusión y llevan a trabajar "a la defensiva".

Estas tres circunstancias en una profesión per se estresante, se convierten en un "caldo de cultivo" para la aparición de desgaste laboral ya que trabajar en esas condiciones puede resultar agotador.

Casi un cuarto de la muestra está afectada por el Síndrome de Desgaste Laboral, el cual se asoció con Satisfacción Laboral Total, Intrínseca y Extrínseca. 
Con niveles medios de cansancio emocional y despersonalización los enfermeros participantes del estudio alcanzan altos niveles de realización personal. El Cansancio Emocional y la Despersonalización se asociaron de manera negativa a Satisfacción Laboral, mientras que la Realización Personal depende directamente del nivel de Satisfacción Laboral.

Con respecto al síndrome de desgaste laboral, los profesionales encuestados con niveles medios de cansancio emocional y despersonalización, obtuvieron alta realización personal, estos resultados son similares a los obtenidos por Grau $(2006)^{(7)}$ y Pera (2002) ${ }^{(9)}$.

Otros estudios, Albaladejo (2004) ${ }^{(10)}$ y Atance (1997) $)^{(5)}$, asignan un total para Burnout sumando las tres subescalas de las componentes del mismo, cada una con una puntuación y sentido de interpretación distinto, por lo que no se considera correcta esa suma y sus resultados no son comparables con este estudio.

Con respecto a las variables socio demográficas, se encontró que no tener pareja ni hijos son predictivas para cansancio emocional y burnout (Puialto Durán, MJ et al 2006; Marrero Santos; 2003)(11,12). Esto probablemente se deba a la falta de contención que brinda la familia, ya que aquellos que no la tienen, tienden a implicarse excesivamente con su trabajo aumentando las probabilidades de tener burnout.

Algunos estudios mencionan como variables de riesgo, el sexo femenino y turnos rotativos para la despersonalización, en tanto que en otros resultó de riesgo el sexo masculino. En éste estudio y en el de Parada et al $(2005)^{(13)}$ que es el más similar en su metodología, la variable de riesgo para despersonalización fue la falta de Satisfacción Laboral.

Atance Martinez (1997) ${ }^{(5)}$ describe como factores de riesgo para la realización personal, ser mayor de 44 años, estar separado y tener una antigüedad profesional mayor a 19 años, en cambio para esta muestra resultaron predictoras ser del interior de la provincia de Córdoba, trabajar en dependencias provinciales, en menor grado municipales y la falta de satisfacción laboral tal como se mencionara antes.

En síntesis, el perfil de riesgo para $\mathrm{BO}$ o alguno de sus tres componentes en esta muestra es: ser mayor de 40 años, no tener hijos ni pareja, vivir en el interior, no estar satisfechas laboralmente y trabajar en hospitales provinciales o municipales en menor grado.

\section{BIBLIOGRAFÍA}

1. MASLACH, C. Burned out. Human Behavior 1976, 9: 16-22.

2. JOFRE V, VALENZUELA S. Burnout en personal de enfermería de la Unidad de Cuidados Intensivos Pediátricos. Rev Aquichan 2005; 5 (1): 56-63.

3. WAR P, COOK J, WALL T. Scales for the measurement of some work attitudes and aspects of psychological well-being. Journal of Occupational Psychochology 1979; 52: 11-28.

4. MASLACH C, JACKSON S. E. The measurement of experienced burnout, Journal of Occupational Behavior 1981; 2: 99 - 113

5. ATANCE MARTINEZ J. Aspectos Epidemiológicos del síndrome de burnout en personal sanitario. Rev Esp Salud Publica 1997, 71 (3): 293-303.

6. DEL CAMPO M, FERNANDEZ-REPETO VALLS E, MARTINEZ DELGADO J, ROJAS BLANCO A. El síndrome de quemarse por el trabajo (burnout) en los profesionales de enfermería de las unidades de diálisis de la provincia de Cádiz. Revista de la Sociedad Española de Enfermería Nefrológica 1999. Valencia, España [online]. 1999. [citado 10/09/2008] Disponible en:

http://www.revistaseden.org/files/El\%20sindrome\%20de\%20quemarse\%20por\%20el\%20trabajo\%20en $\% 20$ los\%20profesionales\%20de\%20enfermer\%C3\%ADa\%20de\%201as\%20unidades\%20de\%20di\%C3\%A1lisis\%20 de\%20la\%20provincia\%20de\%20C\%C3\%A1diz.pdf

7. GRAU A, SUÑER R, GARCÍA M, en nombre del Grupo de Estudio del Síndrome de Desgaste Laboral en los Hospitales de Girona. Desgaste profesional en el personal sanitario y su relación con los factores personales y ambientales. Gac Sanit. 2006; 19 (6): $463-70$. 
8. LOPEZ FRANCO M, RODRIGUEZ NUÑEZ M, FERNANDEZ SANMARTIN S, MARCOS A, MARTINON TORRES F, MARTINON SANCHEZ J. Síndrome de desgaste profesional en el personal asistencial pediátrico. An Pediatr (Barc) 2005; 62 (3): 248-51

9. PERA G, SERRA-PRAT M. Prevalencia del Síndrome del quemado y estudio de los factores asociados en los trabajadores de un hospital comarcal. Gac Sanit 2002; 16 (6): 480-486

10. AlBALADEJO R, VILlANUEVA R, ORTEGA Síndrome de bournout en el personal de enferemeria en un hospital de Madrid. Revista Española de Salud Publica 2004; 78 (4): 505-516.

11. PUIALTO DURAN MJ, ANTOLIN RODRIGUEZ R, MOURE FERNANDEZ L. Prevalencia del síndrome del quemado y estudio de factores relacionados en las enfermeras del CHUVI (Complexo Hospitalario Universitario de Vigo). Enfermería Global. Revista electrónica semestral de enfermería. 2006; 8. [citado: 10/09/2008]. Disponible en:<http:// www.um.es/eglobal/article/view/320/300 >

12. MARRERO SANTOS L. Burnout en enfermeros que laboran en los servicios de Neonatología de Ciudad de La Habana. Rev Cubana Salud Trabajo 2003; 4: 1-2.

13. PARADA M, MORENO R, MEJIAS Z, RIVAS A, RIVAS F, CERRADA J et al. Satisfacción laboral y síndrome de burnout en el personal de enfermería del Instituto autónomo Hospital Universitario Los Andes (IAHULA), Mérida, Venezuela, 2005. Revista Facultad Nacional de Salud Pública 2005; 23 (1): 33-45.

14. BRESÓ ESTEVE E, SALANOVA M, SCHAUFELI W. Síndrome de estar quemado por el trabajo "burnout" (III): Instrumento de medición. Ministerio de Trabajo y Asuntos Sociales. España. Instituto Nacional de Seguridad e Higiene en el Trabajo. Centro Nacional de Condiciones de Trabajo. NTP 732 2008. [citado 10/09/2008]Disponible en: <http://iaprl.asturias.es/opencms/es/instituto/riesgos_laborales/ergonomia/ergo03/ergo03.html>

15. CANTERA LOPEZ FJ. Evaluación de la satisfacción laboral: métodos directos e indirectos. Ministerio de Trabajo y Asuntos Sociales. España. Instituto Nacional de Seguridad e Higiene en el Trabajo. Centro Nacional de Condiciones de Trabajo. NTP 212 (2003). [citado 06/11/2006] Disponible en: < http://bibliotecavirtual. clacso.org.ar/ar/libros/cuba/.../60ZLC1120.pdf>

16. CHIAVENATO I. Introducción a la Teoría General de la Administración. $4^{\circ}$ Edición. México: MC Graw Hill; 1995.

17. FERNANDEZ LARRAGUIBEL B, Paravic Klijn T. Nurse's job satisfaction in public and private hospital of Province of Concepción, Chile. Ciencia y Enfermería 2003; IX (2): 57-66.

18. FIDALGO VEGA M. Síndrome de estar quemado o "burnout" (I): definición y proceso de generación. Ministerio de Trabajo y Asuntos Sociales. España. Instituto Nacional de Seguridad e Higiene en el Trabajo. Centro Nacional de Condiciones de Trabajo. NTP 704 (2006). [citado06/11/2006] Disponible en: http://iaprl. asturias.es/opencms/es/instituto/riesgos_laborales/ergonomia/ergo03/ergo03.html>

19. FIDAlgo VEGA M. Ministerio de Trabajo y Asusntos Sociales. España. Instituto Nacional de Seguridad e Higiene en el Trabajo Centro Nacional de Condiciones de Tabajo. NTP 705. Síndrome de estar quemado por el trabajo "burnout" (II): consecuencias, evaluación y prevención. [citado 06/11/2006] Disponible en: http:// iaprl.asturias.es/opencms/es/instituto/riesgos_laborales/ergonomia/ergo03/ergo03.html >

20. GIL-MONTE P. El síndrome de quemarse por el trabajo (Síndrome de Burnout) en profesionales de enfermería. Revista Eletrônica InterAção Psy 2003; 1 (1): 19 - 33.

21. LÓPEZ SORIANO F, BERNAL L. Prevalencia y factores asociados con el síndrome de burnout en enfermería de atención hospitalaria. Rev Calidad Asistencial 2002; 17 (4):201-205.

22. MASLACH C, JACKSON S, LEITER M. Maslash Burnout Inventory. Third Edition. Palo Alto CA: Consulting Psychologists Press; 1996.

23. PEREZ BILBAO J. Satisfacción Laboral: escala general de satisfacción. Ministerio de Trabajo y Asuntos Sociales. España. Instituto Nacional de Seguridad e Higiene en el Trabajo. Centro Nacional de Condiciones de Trabajo. NTP 394. [citado 06/11/2006]. Disponible en:< www.insht.es/InshtWeb/Contenidos/.../NTP/.../ ntp_394.pdf>

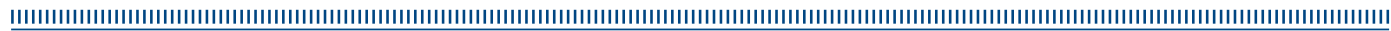

